\title{
Similitude en érosion de cavitation : Essais sur une roue de pompe en eau et en sodium
}

\author{
J.M. Dorey, Ingénieur-chercheur \\ T. Rascalou, Ingénieur-chercheur \\ EDF/DER, Dép̂artement Machines, Chatou
}

\section{La prévision de l'érosion par essais sur modèle : une nécessité industrielle}

Le manque de connaissances sur l'érosion de cavitation a des conséquences directes sur le dimensionnement des machines hydrauliques ainsi que sur leur utilisation.

Pour des machines de conception spécifique pour lesquelles le constructeur ne dispose pas de retour d'expérience, le dimensionnement s'effectue sur la base, soit d'une absence totale de cavitation (ce qui peut conduire à un surdimensionnement), soit d'un critère «maison " plus ou moins validé. Or ce dimensionnement, qui influe sur le coût de la machine, a souvent aussi des conséquences sur les installations.

Le cas des pompes primaires sodium des centrales de type surgénérateur est exemplaire: la taille de ces machines, essentiellement liée à la cavitation, se répercute directement sur la taille de la cuve primaire et donc sur le coût global. De plus, la pompe doit pouvoir fonctionner 40 ans sans démontage. L'optimisation de la taille de la pompe et la vérification de l'absence de risque d'érosion constituent donc un sujet de préoccupation majeur pour les concepteurs.

En ce qui concerne l'exploitation, l'utilisateur est souvent amené, pour améliorer la souplesse de ses installations, à élargir les plages de fonctionnement de ses machines. Mais il lui faut alors vérifier qu'il évitera l'érosion de cavitation.
Pour mieux définir les limites d'une machine vis à vis de l'érosion par cavitation, EDF-DER s'est engagée depuis plusieurs années, en collaboration avec la Direction Générale de l'Armement/DRET, ACB/CERG et l'Institut de Mécanique de Grenoble, dans le développement et la mise au point d'une méthodologie qui permette, à partir d'essais sur modèle réduit, de quantifier les risques d'érosion et de prédire la perte de masse sur le prototype.

Les essais sur pompe décrits ci-après s'inscrivent donc dans ce cadre et sont le prolongement d'essais effectués sur veine fixe en mercure par l'IMG, en eau par le CERG, et en sodium par EDF.

\section{La méthodologie utilisée}

Cette méthodologie repose sur une théorie de similitude que l'on doit à Y. LeCOFFre (Réf. [1]). Il nous a semblé opportun de refaire ici un rapide point sur la méthode, ses fondements et l'éclairage qu'apportent les résultats obtenus jusqu'ici.

\subsection{La théorie}

L'érosion de cavitation est générée par une succession d'implosions de structures de vapeur à proximité des

\section{Cavitation erosion scaling : tests on a pump impeller in water and in sodium}

Tests to quantify cavitation agressivity carried out in water and in sodium $\left(400^{\circ} \mathrm{C}\right)$ on a model pump impeller are presented. The polished samples method has been used. It can be now applied to curved surfaces such as impeller blades with the help of new measurement devices. Results are discussed regarding scaling laws for fluid-to-fluid transposition. 
parois. L'existence, la violence et la fréquence de ces événements, sont le résultat de conditions hydrodynamiques données. D'où l'idée qu'avec des lois de similitude hydrodynamique adaptées, il doit être possible de transposer, d'une échelle $L_{1}$ à une autre $L_{2}$, d'un fluide (1) à un autre (2), d'une vitesse $V_{1}$ à l'autre $V_{z}$, les effets de ces implosions.

\section{a) A l'échelle $\mathrm{L}$ de l'écoulement, les similitudes à respecter}

Dans le cas de cavitation par poche attachée (cas des pompes), il faut respecter :

- la similitude géométrique, (géométrie similaire, incidence fluide identique) pour assurer en des points similaires des vitesses réduites (c'est-à-dire rapportées à la vitesse de référence $V_{\text {ref }}$ ) et des coefficients de pression $C_{p}$ égaux ;

— la similitude de Sigma $\left(\sigma=\left(P_{\text {ref }}\left(\right.\right.\right.$ Pvap $\left.-/ 0,5 \rho V r e f^{2}\right)$ qui assure un développement de la cavitation en similitude, notamment des poches de longueur similaires.

La similitude de Reynolds ne pouvant pas, en général, être respectée, il convient au moins de s'assurer par des visualisations que dans les conditions d'écoulement étudiées, son effet est marginal.

\section{b) Les conséquences à l'échelle I des structures de vapeur produites}

Il a été constaté que la fréquence $f$ de production des structures de vapeur derrière une poche suit une loi de Strouhal $\left(S t=f L / V_{0}\right)$ constant. Ainsi, appliquer les similitudes citées ci-dessus assure donc une similitude des structures de vapeur émises en ce qui concerne :

- leurs dimensions, d'échelle caractéristique $I$ proportionnelle à $L$, dimension caractéristique de l'écoulement, et donc dans le rapport d'échelles $\lambda=L_{1} / L_{2}$;

- leur nombre: proportionnel au rapport des vitesses fluides et à $\lambda^{-1}$ (loi de Strouhal constant);

- les conditions d'implosion: la pression subie lors de l'implosion est proportionnelle au carré des vitesses, ce qui conduit à des vitesses en fin d'implosion $V_{i}$ proportionnelles à la vitesse de référence de l'écoulement $V_{r e f}$ (Réf. [9]).

\section{c) Les conséquences pour les impacts subis par la paroi}

La paroi subit des impacts qui résultent des implosions, sous la forme soit d'une onde de pression, soit d'un choc de microjet. Dans les deux cas, l'impact, c'est-à-dire le chargement auquel est soumis le matériau, peut être caractérisé par trois parenthèses :

- la pression $P_{i}$ qui s'applique sur la paroi, et qui est proportionnelle à $\rho c V_{i}$, donc à $\rho c V_{r e f}, c$ étant la célérité du son dans le fluide et $\rho c$ son impédance acoustique;

- la durée $\Delta t_{i}$ pendant laquelle cette pression s'applique, qui doit être proportionnelle à $l / c$;

- une dimension $d_{i}$, caractéristique de la surface sur laquelle elle s'applique, et proportionnelle à $l$, donc à $L$.
L'ensemble des impacts n'est en réalité pas uniforme et doit donc être décrit par une répartition statistique en fonction de la pression de la durée et de la taille. C'est cette répartition que l'on appellera par la suite histogramme d'impacts et qui caractérise l'agressivité.

\section{d) Lois de transposition}

Les assertions précédentes permettent donc d'établir les règles suivantes :

- d'une échelle à l'autre, à même vitesse d'écoulement, les impacts résultants de structures similaires ont des dimensions caractéristiques dans le rapport des échelles $\lambda$ et des pressions égales. Quant à leurs nombres respectifs, ils sont dans le rapport inverse des échelles. Autrement dit, les taux d'impacts par unité de surface sont dans le rapport $\lambda^{-3}$. Les durées d'impacts similaires sont alors dans le rapport $\lambda$;

- d'une vitesse à l'autre, les impacts similaires ont des pressions et des fréquences dans le rapport des vitesses $V_{1} / V_{2}$. Les durées d'impact et leurs tailles restent quant à elles inchangées ;

- d'un fluide à l'autre, avec des vitesses d'écoulement dans le rapport inverse des impédances acoustiques des fluides $(\rho c)_{2} /(\rho c)_{1}$, les pressions d'impacts similaires sont égales, leurs tailles sont égales, leurs durées sont dans le rapport des célérités du son $c_{1} / c_{2}$. Leur nombre est quant à lui proportionnel au rapport des vitesses.

Ces règles permettent donc, en théorie, de déduire de l'histogramme d'impacts sur modèle à vitesse réduite, en fluide différent, celui auquel sera soumis le prototype (Réf. [1]).

\subsection{La mesure par échantillons polis}

La mesure se fait en insérant des échantillons finement polis à la paroi soumise à l'érosion. Après une courte exposition à la cavitation, on relève les indentations obtenues. Chacune peut être caractérisée par un diamètre $d_{\mathrm{t}}$ et une profondeur $h_{\mathrm{t}}$. Des moyens de dépouillement récemment développés (systèmes interférométriques ou profilomètres laser) permettent de déterminer ces grandeurs. On peut ainsi établir un histogramme donnant le nombre de marque par classe de tailles.

En première approximation, il a été postulé que le matériau réagissait comme un filtre: il ne se marque que pour des impacts de pression supérieure à une valeur seuil caractéristique du matériau. Ainsi l'histogramme des marques obtenu est l'image d'une partie de l'histogramme d'impacts, limitée par cette valeur seuil.

En outre, l'utilisation dans les essais modèle d'un matériau plus ductile (à seuil bas) devrait permettre d'obtenir l'histogramme prototype à partir d'essais à vitesse réduite.

L'histogramme obtenu peut alors être transposé au prototype à vitesse réelle sur matériau dur (seuil élevé). Le rapport des seuils fixe le rapport des vitesses à respecter. 


\subsection{La prévision de la perte de masse}

Une fois l'agressivité mesurée, puis transposée au prototype, la vitesse d'érosion sur le prototype doit être quantifiée. Pour ce faire, $\mathrm{ACB} / \mathrm{CERG}$ a développé un appareil de reproduction d'impacts en laboratoire, (CAVERSIM), basé sur l'implosion d'un filament de vapeur au sein d'un vortex entretenu. Il est actuellement en phase de calibration (Réf. [8]).

\subsection{Un point sur la validation}

Un premier ensemble de campagnes d'essais avait été réalisé dans le courant des années 80, toujours en collaboration entre la DGA/DRET, EDF, ACB/CERG et l'IMG. Une géométrie commune d'essai a été définie, une valeur de $\sigma$ a été fixée, et plusieurs campagnes ont été engagées :

- en eau à échelles 1 et 3 (ACB/CERG). Vitesses comprises entre $20 \mathrm{~m} / \mathrm{s}$ et $50 \mathrm{~m} / \mathrm{s}$ (Réf. [1 et 4]);

- en mercure à échelles 1 et $1 / 3$ (IMG). Vitesses allant jusqu'à $8 \mathrm{~m} / \mathrm{s}$ (Réf. [2 et 7]);

— en sodium à échelle 1 à $300 / 400 / 500^{\circ} \mathrm{C}$ (EDF). Vitesses comprises entre $25 \mathrm{~m} / \mathrm{s}$ et $40 \mathrm{~m} / \mathrm{s}$ (Réf. [4]).

Nous ne reprendrons pas ici l'analyse des résultats, mais on peut rappeler certaines constatationss essentielles :

1. Les lieux d'érosion maximale sont similaires dans tous les cas, quels que soient l'échelle et le fluide. Ceci confirme notamment le bien-fondé des similitudes hydrodynamiques et de Sigma.

2. Lorsqu'elles ont été enregistrées, les fréquences de fluctuations suivent bien une loi de Strouhal constant.

3. En mercure, pour les plus fortes vitesses, le nombre d'indentations est proportionnel à la vitesse. C'est un résultat très important, puisqu'il montre que la production d'impacts est bien, elle aussi, proportionnelle à la vitesse.

4. Entre une échelle et une autre, la taille des indentations évolue bien dans le même ordre que le rapport des échelles.

Cependant, tous les résultats ne vérifient pas directement les lois émises. En particulier il semble qu'entre deux échelles, la loi du $\lambda^{3}$ ne soit pas totalement respectée (Réf. [4 et 7]). D'un fluide à l'autre, la loi de transposition ne paraît pas non plus bien confirmée.

En ce qui concerne la comparaison eau/sodium, le rapport des impédances acoustiques est de 1,38 pour une température du sodium de $400^{\circ} \mathrm{C}\left(\rho=857 \mathrm{~kg} / \mathrm{m}^{3}, c=\right.$ $2365 \mathrm{~m} / \mathrm{s}$ ). Ainsi en théorie, un essai de marquage en eau à une vitesse 1,38 fois plus importante qu'en sodium doit donner un taux de marques 1,38 fois plus important.

Dans le tableau ci-après, on montre les résultats d'essais en sodium $\left(400^{\circ} \mathrm{C}, \mathrm{V}=28.5 \mathrm{~m} / \mathrm{s}\right.$ et $\left.39,4 \mathrm{~m} / \mathrm{s}\right)$ et en eau $(\mathrm{V}=40 \mathrm{~m} / \mathrm{s}$ et $50 \mathrm{~m} / \mathrm{s})$ théoriquement presque comparables. Les dépouillements réalisés par l'IMG (Réf. [5]) ont donné les valeurs des taux de marques et taux de volume déformé pour diverses classes de tailles de marques.

\begin{tabular}{|c|c|c|c|c|c|c|c|c|}
\hline Fluide & \multicolumn{4}{|c|}{ Sodium } & \multicolumn{4}{|c|}{ Eau } \\
\hline Vitesse & \multicolumn{2}{|c|}{$28.5 \mathrm{~m} / \mathrm{s}$} & \multicolumn{2}{|c|}{$39,4 \mathrm{~m} / \mathrm{s}$} & \multicolumn{2}{|c|}{$40 \mathrm{~m} / \mathrm{s}$} & \multicolumn{2}{|c|}{$50 \mathrm{~m} / \mathrm{s}$} \\
\hline $\begin{array}{l}\text { Tailles des } \\
\text { marques } \\
(\mu \mathrm{m})\end{array}$ & $\begin{array}{l}\text { Taux de } \\
\text { marques } \\
\left(\mathrm{n} / \mathrm{cm}^{2} / \mathrm{s}\right)\end{array}$ & $\begin{array}{l}\text { Volume } \\
\text { deforme } \\
4 \mathrm{~m}^{3} / \mathrm{cm}^{2} / \mathrm{s}\end{array}$ & $\begin{array}{l}\text { Taux do } \\
\text { marques } \\
\left(n / \mathrm{cm}^{2} / \mathrm{s}\right)\end{array}$ & $\begin{array}{l}\text { Volume } \\
\text { deforme } \\
\mu \mathrm{m}^{3} / \mathrm{cm}^{2} /\end{array}$ & $\begin{array}{l}\text { Taux de } \\
\text { marques } \\
\left(\mathrm{n} / \mathrm{cm}^{2} / \mathrm{s}\right)\end{array}$ & $\begin{array}{l}\text { Volume } \\
\text { ceforme } \\
\text { im } \mathrm{m}^{3} / \mathrm{cm}^{2} / \mathrm{s}\end{array}$ & $\begin{array}{l}\text { Taux de } \\
\text { marques } \\
\left(\mathrm{n} / \mathrm{cm}^{2} / \mathrm{s}\right)\end{array}$ & $\begin{array}{l}\text { Volume } \\
\text { defforme } \\
\mu^{3} / \mathrm{cm}^{2} /\end{array}$ \\
\hline $20-30$ & 0.531 & 16,0 & 0.974 & 34,5 & 2,09 & 44,1 & 7,20 & 109 \\
\hline $30-40$ & 0.531 & 34,4 & 0.779 & 47,7 & 1,87 & 101,2 & 5,65 & 240 \\
\hline $40-60$ & 0,598 & 34,9 & 0,866 & 114,6 & 2,96 & 326 & 8,348 & 939 \\
\hline $60-100$ & 0,548 & 486 & 0.952 & 640 & 1,868 & 705,2 & 8,452 & 4179 \\
\hline $100-1$ & 0,205 & 465 & 0.390 & 969.5 & 1,260 & 3075 & 2,50 & 4294 \\
\hline $150-300$ & 0,133 & 2331 & 0.347 & 3453 & 0.392 & 2761 & 2,19 & 24817 \\
\hline $300-600$ & 0,010 & 333 & 0,087 & 5367 & 0,044 & 888 & 0,207 & 14316 \\
\hline Total & 2,556 & 3700 & 4,395 & 10625 & 10,484 & 5167 & 34,547 & 48894 \\
\hline
\end{tabular}

Tout d'abord, cet exemple montre bien que le nombre (taux) total de marques n'est pas en soi représentatif puisque les marques de petites tailles (inférieures à $60 \mu \mathrm{m}$ ), qui sont les plus importantes en nombre, ne contribuent que très faiblement au volume déformé total. Cependant, il faut bien admettre qu'à vitesse équivalente, pour les marques participant le plus au volume, le rapport est de l'ordre de 2 à 6 , plutôt que de 1,38 .

Parmi les causes possibles des écarts observés, on peut citer :

— des effets d'échelle au sens hydraulique; par exemple l'influence de la turbulence sur la relation entre production de structures de vapeur et production d'impacts, ou entre la dimension $l$ et la distance caractéristique $d_{\mathrm{i}}$;

- la variation de la célérité du son avec la pression ;

— un comportement du matériau plus complexe que celui envisagé ;

- de possibles imprécisions dans la reproduction de la géométrie.

En conclusion, sans que l'on puisse prétendre que la validation soit faite, les concepts de base des lois énoncées semblent confirmés par une série d'essais qui ne portent cependant que sur une seule géométrie. Il nous paraît donc souhaitable de poursuivre les études du phénomène de manière à bien identifier et quantifier les effets d'échelle.

\section{Les essais sur pompe}

\subsection{Objectifs et description des essais}

La campagne d'essai a des objectifs multiples :

- faisabilité technique de l'application de la méthode à un modèle de machine hydraulique ;

- validation de la transposition eau/sodium;

- détermination de la courbe de NPSH limite d'érosion à une vitesse de rotation fixée.

Rappelons l'application des similitudes à une pompe :

- entre modèle et prototype, on obtient des vitesses fluides égales pour des vitesses de rotation dans le rapport inverse des échelles : $N_{\text {mod }} / N_{\text {prot }}=L_{\text {prot }} / L_{\text {mod }}=\lambda^{-1}$; 


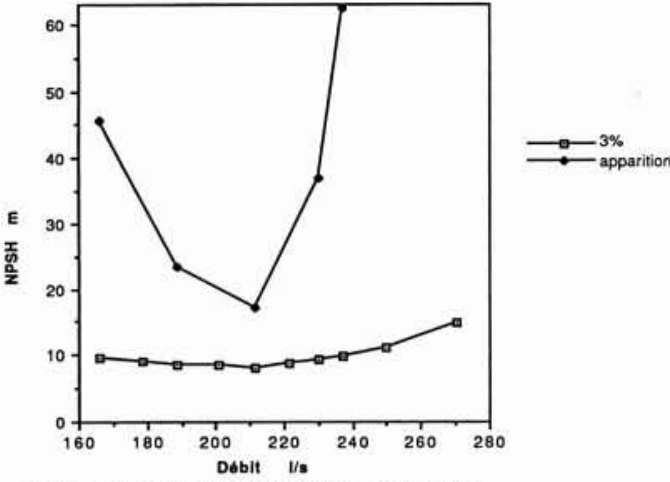

Fig 1: NPSH $3 \%$ ot NPSH d'apparition de la pompe

on fonction du debit 2750 trimn

1. NPSH $3 \%$ et NPSH d'apparition de la pompe en fonction $d u$ débit à $2750 \mathrm{tr} / \mathrm{mn}$.

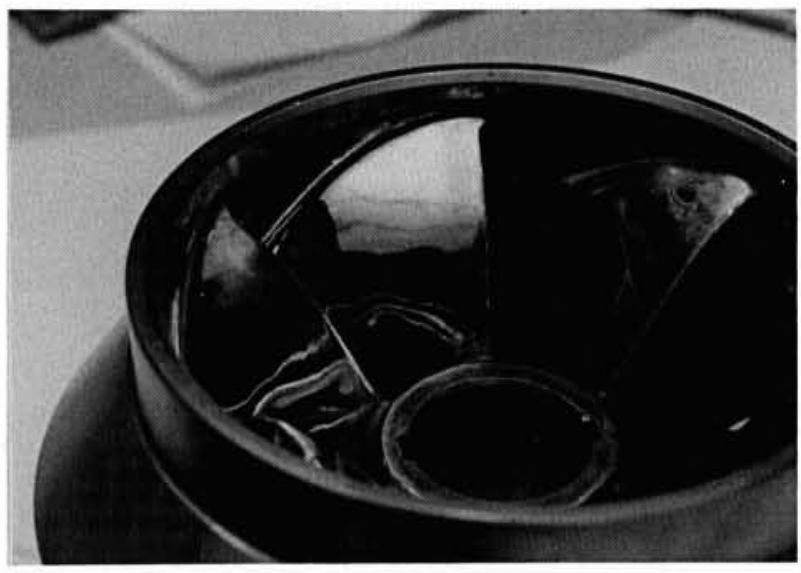

2. Photo de la roue polie.
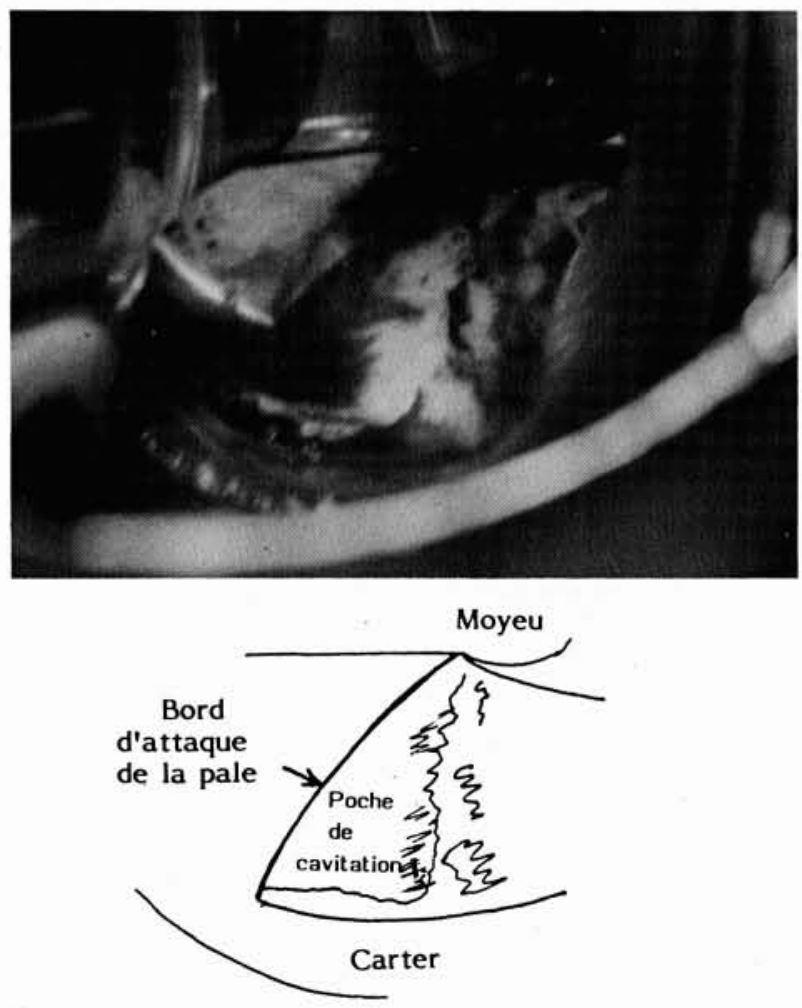

3. Poche de cavitation sur une pale lors de l'essai en eau à $3300 \mathrm{tr} / \mathrm{mn}$

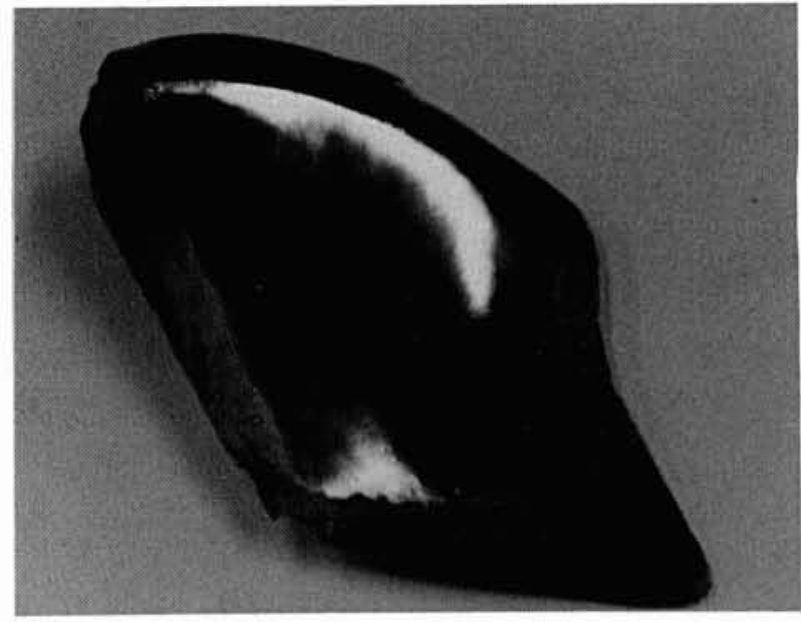

4. Empreinte métallisée.

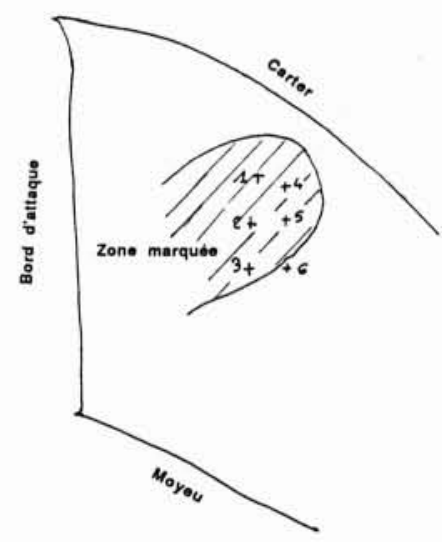

5. Zone d'érosion relevée sur les pales après l'essai Sodium. Localisation des points de mesure.

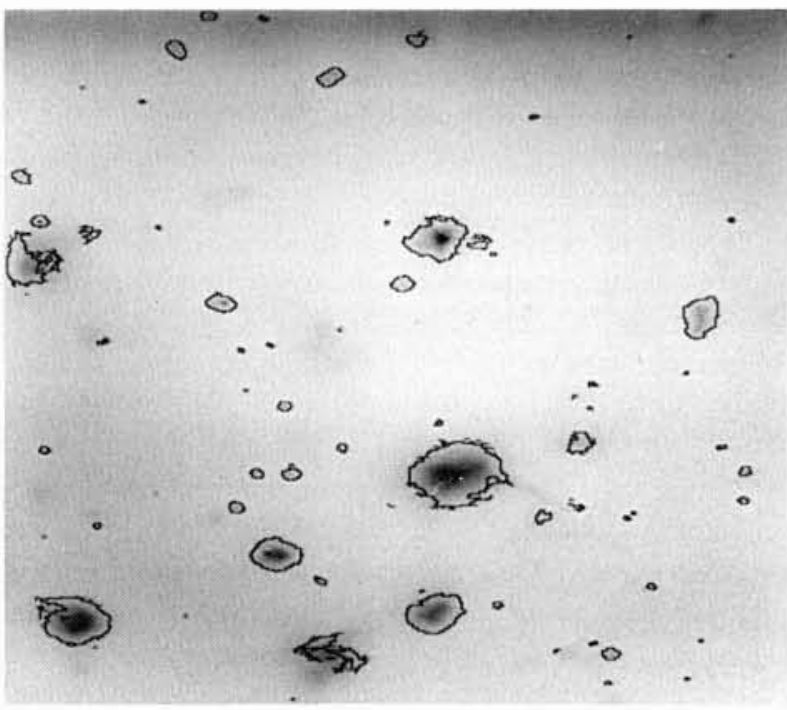

6. Résultat de l'analyse de marques par traitement d'image. 
- le sigma se calcule par la formule : $\sigma=g(N P S H)$ | $0,5 U_{1}^{2}, U_{1}$ étant la vitesse périphérique en entrée ;

- une même incidence fluide implique de conserver le coefficient de débit $\phi=Q / \pi \omega R^{3}$, (avec $\omega=2 \pi \mathrm{N} / 60$ et $\mathrm{R}$ rayon d'entrée de la roue) ou plus prosaïquement le rapport $Q / Q n$, où $Q n$ est le débit nominal, proportionnel à la vitesse de rotation $N$.

La première étape de la campagne consiste à déterminer le coefficient de vitesse à respecter entre les deux fluides. Le résultat d'un essai en sodium est comparé à ceux de plusieurs essais en eau.

Une fois la vitesse en eau fixée, on effectue des essais, pour différents NPSH/débits. L'analyse des marquages obtenus devra permettre, à l'aide notamment du CAVERSIM, de déterminer les taux d'érosion correspondants. On en déduira ainsi une courbe de NPSH de début d'érosion en fonction du débit pour le prototype.

\subsection{Description du matériel}

La pompe est un modèle à l'échelle 0,209 de la pompe primaire envisagée dans le projet SPX2. Elle est de type hélicocentrifuge, avec une entrée radiale, un diffuseur aubé et une sortie axiale.

Ses principales caractéristiques sont :

Vitesse spécifique: Ns $=57$ au point nominal projet ;

Nombre d'aubes de la roue : 6 ;

Nombre d'aubes du diffuseur : 8 ;

Diamètre roue : $284 \mathrm{~mm}$.

La roue a été réalisée en acier inoxydable $316 \mathrm{~L}$ par moulage en cire perdue. Tous les essais ont été effectués avec la même roue. Par contre les maquettes en eau et en sodium ne sont pas strictement identiques, mais la géométrie du canal d'entrée est respectée dans sa partie essentielle en amont de la roue. Le comportement en cavitation de la pompe à $2750 \mathrm{tr} / \mathrm{mn}$ est sommairement décrit par la figure 1 .

Pour nos essais, nous avons utilisé les deux installations suivantes :

- la boucle CARUSO pour les essais en sodium : c'est une installation du C.E.A., implantée au centre de Cadarache, prévue pour tester des pompes modèles pour des débits allant jusqu'à $300 \mathrm{l} / \mathrm{s}$;

- la boucle EPOCA pour les essais en eau : il s'agit d'une installation EDF/DER implantée à Gennevilliers, spécifiquement conçue pour les essais de pompes en régime cavitant, et permettant notamment de fonctionner avec une eau dégazée.

\subsection{Les procédures de dépouillement utilisées}

Le polissage est effectué directement sur la face visible des aubes de la roue, jusqu'à $50 \mathrm{~mm}$ du bord d'attaque tout en évitant ce dernier (fig. 2). Après essai, on réalise des empreintes en Technovit des zones attaquées. Celles-ci sont ensuite métallisées sous vide (fig. 3). Ce procédé introduit cependant des ondulations parasites assez régulières de l'ordre de $40 \mu \mathrm{m}$, qu'il faut filtrer lors du dépouillement. La mesure sur les empreintes se fait à l'aide d'un profilomètre laser de marque UBM équipé de tables de déplacement $X$ et $Y$, le tout étant piloté par un microordinateur PC. On obtient ainsi une côte $Z$ en chaque point scruté par le faisceau laser. Ici, compte tenu de la taille des marques, nous avons choisi de traiter des surfaces de $2 \mathrm{~mm} \times 2 \mathrm{~mm}$ à raison de un point tous les $5 \mu \mathrm{m}$, soit des fichiers de 400 $\times 400$ points.

Ensuite, par calcul, on dégauchit la surface en soustrayant la surface moyenne, puis on multiplie le résultat par -1 pour obtenir la surface impactée. Pour supprimer le bruit introduit, le fichier est filtré.

Pour ensuite extraire les impacts, nous utilisons un procédé de traitement d'image : le fichier surface est transformé en image et un logiciel spécifique permet de localiser et identifier les impacts (fig. 6), puis de quantifier la géométrie de chacun : taille, profondeur, volume.

A partir du fichier résultat, on peut établir des histogrammes. Dans un premier temps, nous avons extrait la valeur $V$ qui représente la somme des volumes d'impacts, divisée par le temps et la surface, que nous appelons taux de volume déformé. Bien que insuffisante pour décrire l'agressivité, cette grandeur paraît cependant assez significative.

\subsection{Résultats}

\section{a) En sodium}

En sodium, un seul essai sur roue polie a pu être effectué, dans les conditions suivantes:

Vitesse : $2750 \mathrm{tr} / \mathrm{mn}$;

NPSH : $11,5 \mathrm{~m}$;

Température : $400^{\circ} \mathrm{C}$;

Débit : $1901 / \mathrm{s}$, soit $0,8 \mathrm{Qn}$;

Durée : $1 \mathrm{~h}$.

Lors des essais, des problèmes de mesure ont engendré une in certitude importante (de l'ordre de $\pm 5 \%$ ) sur le débit.

De l'observation de la roue, on retire les constatations suivantes :

- toutes les pales sont marquées ;

- la localisation des érosions est comparable d'une pale à l'autre (cf. fig. 5);

- les impacts significatifs sont de l'ordre de $150 \mu \mathrm{m}$ de diamètre.

Le dépouillement a été effectué sur les pales 1 et 4 , en 5 points par pale (soit $20 \mathrm{~mm}^{2}$ ) et les fichiers (environ 100 marques par point) ont été additionnés.

On obtient ainsi un taux de volume déformé $V$ de : $11,2 \mu \mathrm{m}^{3} / \mathrm{mm}^{2} / \mathrm{s}$ sur la pale 1 et de $10,9 \mu \mathrm{m}^{3} / \mathrm{mm}^{2} / \mathrm{s}$ sur la pale 4 .

\section{b) En eau}

En eau, les essais sont réalisés dans des conditions similaires à celles de l'essai en sodium. Pour le premier essai, 
la vitesse est fixée à 1,2 fois la vitesse sodium, compte tenu des résultats des essais " veine fixe ", soit $3300 \mathrm{tr} / \mathrm{mn}$. Par contre, l'installation ne permet pas actuellement d'aller au-delà de $3518 \mathrm{tr} / \mathrm{mn}$, soit un coefficient de 1,28 . Successivement, les essais suivants sont effectués :

- essai à $3300 \mathrm{tr} / \mathrm{mn}, 0,8 \mathrm{Qn}, \mathrm{NPSH}=16,5 \mathrm{~m}$, durée $1 \mathrm{~h}$ : aucun marquage visible ;

- essai à $3518 \mathrm{tr} / \mathrm{mn}, 0,8 \mathrm{Qn}, \mathrm{NPSH}=20 \mathrm{~m}$, durée $1 \mathrm{~h}$ : les pales 1 et 6 sont faiblement marquées. Cependant, la localisation des marquages ne correspond pas exactement à celle observée après l'essai en sodium. Ceci est confirmé par un essai à même vitesse, même débit, $\mathrm{NPSH}=18,8 \mathrm{~m}$.

Compte tenu de l'incertitude sur le débit sodium, des essais à $0,75 \mathrm{Qn}$ ont été réalisés.

- essai à $3518 \mathrm{tr} / \mathrm{mn}, \mathrm{NPSH}=18,8 \mathrm{~m}$, durée $1 \mathrm{~h} 30$ : toutes les pales sont marquées, la localisation correspondant à l'essai sodium, mais l'intensité est plus faible. Le taux de volume déformé $V$ vaut $3,5 \mu \mathrm{m}^{3} / \mathrm{mm}^{2} / \mathrm{s}$ sur la pale 1 ;

- essai à $3300 \mathrm{tr} / \mathrm{mn}, \mathrm{NPSH}=16,5 \mathrm{~m}$, durée $3 \mathrm{~h}$ : seules les pales 1,4 , et 6 sont sensiblement marquées. Le taux de volume déformé $V$ vaut $1,13 \mu \mathrm{m}^{3} / \mathrm{mm}^{2} / \mathrm{s}$ sur la pale 1 .

Il apparaît certain que l'agressivité a été nettement plus forte en sodium. Si l'on extrapole les deux derniers résultats en eau, on obtient qu'il faudrait fonctionner à $3900 \mathrm{tr} / \mathrm{mn}$ pour obtenir une agressivité comparable au sodium (cf. fig. 7). Ainsi, le coefficient entre sodium et eau serait de l'ordre de 1,4, donc beaucoup plus proche de la valeur théorique.

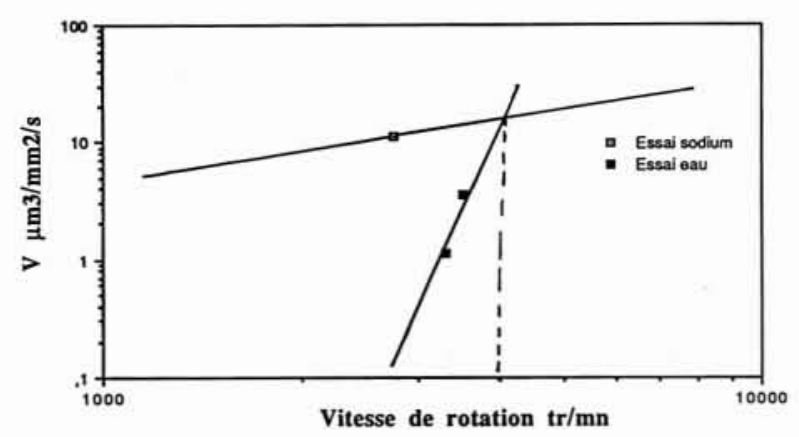

7. Taux de volume déformé en fonction de la vitesse de rotation en eau à 0,75 Qn et comparaison au résultat en sodium.

En ce qui concerne la validation des lois de transposition, il se confirme que le sodium est nettement plus agressif que l'eau, ce qui avait déjà été observé par d'autres. Ainsi, le rapport entre les vitesses en eau et en sodium nécessaires pour obtenir des agressivités comparables a été trouvé de l'ordre de 1,40 ce qui est très proche du rapport des impédances acoustiques prévu par la théorie mais qui est relativement différent du résultat des essais précédents de comparaison eau/sodium sur veine fixe. Cependant, ce résultat basé sur un seul essai en sodium nécessitera d'autres confirmations.

\section{Conclusions}

La similitude proposée par Y. LECOFFRE en matière d'érosion par cavitation nous parait aujourd'hui être suffisamment confirmée pour permettre d'aller de l'avant dans la méthodologie de prévision à partir d'essais sur modèle réduit. Certains écarts observés sur les premiers essais nous semblent être imputables à des effets d'échelle qui doivent être étudiés, si possible en utilisant d'autres géométries, mais ne remettent pas en cause le fondement de la théorie.

Les essais effectués sur pompe constituent la première tentative de mesurer sur une machine modèle l'agressivité de l'érosion de cavitation par la méthode du marquage de surfaces polies.

La méthode s'est révélée efficace. Les adaptations nécessaires (polissage de surfaces gauche, dépouillement par répliques et procédures de mesure des indentations...) ont été éprouvées et permettent d'obtenir des résultats tangibles. Cependant, sa mise en œuvre reste délicate.

Les améliorations envisagées pour aboutir à une méthode à la fois plus sûre et plus souple concernent :

- la précision géométrique du modèle : des écarts substantiels d'agressivité ont été observés entre les pales pour des écarts géométriques faibles. Les méthodes de fabrication de ces modèles doivent donc atteindre, relativement à l'échelle, la précision des machines prototypes, voire mieux ;

- l'utilisation de matériaux plus mous permettant de travailler à des vitesses plus faibles reste encore à étudier.

\section{Références}

[1] Y. Lecoffre, J. Marcoz, G. Maisonnier. - Erosion de cavitation, étude théorique et expérimentale des lois de similitude. Rapport CERG/DRET, juillet 1984.

[2] J.P. Franc, J.M. Michel, L. Treillard. - Similitude en érosion de cavitation; essai en mercure. Rapport IMG/DRET, septembre 1984.

[3] J.M. DOREY. - Similitude en érosion de cavitation; essais en sodium. Rapport EDF, juillet 1986.

[4] S. Lavigne. - Description d'une méthode de prévision de l'érosion de cavitation. Essais d'érosion à l'eau. Communications lors de la Journée Thématique Cavitation DGA/DRET à Arcueil le 13 juin 1991

[5] J.L. Reboud, R. Fores-Patella, J.M. Dorey. - Simulation of cavitation impact damage on an elastoplastic solid. Cavitation and multiphase flow Forum ASME/JSME, Portland, 23-27 juin 1991.

[6] B. Belahadj, O. Blottiaux, J.P. Franc, J.P. Franc, J.M. MICHEL. - Analyse statistique d'impacts d'érosion de cavitation. Rapport IMG/EDF, octobre 1989.

[7] J.P. Franc, J.M. Michel, H. Nguyen Trong. - Effet d'échelle géométrique en érosion de cavitation : essais d'érosion sur boucle à mercure. Rapport IMG/DRET septembre 1991.

[8] A. AMBlaRd. - Etude de réalisation du Carversim. Rapport CERG/EDF 1989.

[9] Y. Lecoffre, P. Grison, J.M. Michel. - Prévision de l'érosion de cavitation pour les turbomachines. AIRH, Montréal, septembre 1986. 